\title{
QCD and a new paradigm for nuclear structure
}

\author{
A. W. Thomas ${ }^{1, a}$ \\ ${ }^{1}$ CSSM and ARC Centre of Excellence for Particle Physics at the Terascale, \\ School of Chemistry and Physics, University of Adelaide, Adelaide SA 5005, Australia
}

\begin{abstract}
We review the reasons why one might choose to seriously re-examine the traditional approach to nuclear theory where nucleons are treated as immutable. This examination leads us to argue that the modification of the structure of the nucleon when immersed in a nuclear medium is fundamental to how atomic nuclei are built. Consistent with this approach we suggest key experiments which should tell us unambiguously whether there is such a change in the structure of a bound nucleon. We also briefly report on extremely promising recent calculations of the structure of nuclei across the periodic table based upon this idea.
\end{abstract}

\section{Introduction}

Since the discovery of the neutron in the 1930s, the overwhelming majority of theoretical studies of nuclear structure have adopted the hypothesis that the protons and neutrons inside a nucleus are immutable objects whose internal structure never changes. These immutable objects interact through non-relativistic two- and three-body forces and the challenge is primarily to accurately solve the many-body problem. The phenomenological forces used include physics such as Yukawa's pion exchange and as a consequence the precise calculation of observables may require the inclusion of exchange current corrections.

Beginning with the famous one-boson-exchange potentials [1], it became clear that the dominant part of the intermediate range attraction between nucleons had a Lorentz scalar, isoscalar character, which was phenomenologically represented by the exchange of a $\sigma$ meson. For decades this meson was viewed as an artifact involving an unphysical meson used purely for convenience. However, careful dispersion relation treatments of $\pi N$ scattering in the past decade have shown that this state does indeed exist [2]. Confirmation of this Lorentz scalar, isoscalar character of the intermediate range attraction in the $N N$ force also came from dispersion relation studies by groups in Paris [3], Stony Brook and elsewhere. Walecka and coworkers exploited the Lorentz scalar nature of the $N N$ attraction and the Lorentz vector character of the short range repulsion to build a very successful, fully relativistic theory of nuclear matter [4] and later finite nuclei [5]. Here too the nucleons were immutable.

All this was very satisfactory but for one vexatious issue. At nuclear matter densities the typical mean scalar field strength felt by a bound nucleon in the Walecka model is of order $500 \mathrm{MeV}$. This is a huge number. As

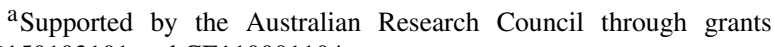
DP150103101 and CE110001104.
}

a consequence, the effective mass of the bound nucleon is only one half of its free mass.

At around the same time as Walecka and collaborators developed their model, the theory of the strong interaction underwent a revolution. Quantum Chromodynamics was developed as a local gauge field theory built on color. It became clear that, by analogy with Rutherford's work on the nucleus within the atom, the natural explanation of the discovery of scaling at SLAC in the late 60's [6] was that the nucleon too was primarily empty space containing point-like quarks.

From this more fundamental point of view the huge scalar field experienced by a bound nucleon is even more challenging. How can it be that the exchange of a scalar meson, which must couple to the confined quarks in the nucleon with such strength, can have no effect on the internal structure of the nucleon, which after all is far from point-like?

Considerations like these led Guichon [7] to propose a dramatically different approach to nuclear binding, the Quark Meson Coupling (QMC) model, where the effect of the mean scalar field generated by other nucleons is treated self-consistently in solving for the wave function of each confined quark. Taking the simplest form for the coupling of the $\sigma$ and $\omega$ mesons to quarks confined in the MIT bag model [8, 9], means that in nuclear matter the vector field simply shifts the definition of the energy, while the scalar field modifies the Dirac wave function. This difference in the effect of the two Lorentz components of the nuclear mean field is crucial, as their effects more or less cancel when it comes to the total energy but for the quark motion (or loosely speaking, wave function) the scalar field is not cancelled.

A critical effect of the change in the quark wave function induced by an attractive scalar field is that the size of the lower Dirac component increases. In turn this reduces the value of $\int d V \bar{\psi} \psi$, which defines the overall strength 
with which the scalar field couples to the nucleon. This process is completely analogous to the way an atom rearranges its internal structure to oppose an applied electric field. Thus the parameter calculated within any particular quark model which describes this is called the "scalar polarizability", $d$. The overall scalar coupling to the nucleon is written in the simplest approximation as

$$
g_{\sigma N}(\sigma)=g_{\sigma N}(0)-\frac{d}{2}\left(g_{\sigma N}(0) \sigma\right)^{2}
$$

In the MIT bag model $d \approx 0.22 R$, with $R$ the bag radius.

This behaviour is very straightforward and appears in all relativistic quark models used so far. Nevertheless, in terms of nuclear structure it is profound. Whereas the repulsion felt by each nucleon grows linearly with density, the scalar attraction saturates as the density rises and one naturally finds saturation of nuclear matter. This mechanism is both new and extremely effective. As a result the mean scalar field felt by a nucleon at the saturation density of nuclear matter is just a few hundred $\mathrm{MeV}$, much lower than that found in the Walecka model.

Philosophically, this approach is radically different from anything done before because the colourless clusters of quarks which occupy single particle levels in nuclear matter may have nucleon quantum numbers but their internal structure is modified. Almost immediately it was shown [10] that this change could account for the key features of the famous nuclear EMC effect, discovered in the early 80 's.

Later the model was developed further by Guichon, Rodionov and Thomas [11] to correctly treat the effect of spurious centre of mass motion in the bag, which had resulted in anomalously small $\omega N$ couplings. In the same paper the model was also extended to finite nuclei, showing very naturally how one obtains realistic spin orbit forces. Finally, since the model is built at the quark level, using the same quark model, with the same quarkmeson coupling constants, one can derive the properties of any bound hadron. For example, it shows very naturally why the spin-orbit force for the $\Lambda$ hyperon is extremely small $[12,13]$. For a complete overview of the phenomenological consequences of the QMC model we refer to the review by Saito et al. [14].

With the motivation for the QMC approach clearly established, one is naturally led to the following lines of investigation. First, given the success of the conventional approach to nuclear structure based upon non-relativistic two- and three-body forces, it is natural to ask how that is related to QMC. We address this in section 2. Second, one may also ask what evidence there is to support the at first sight radical idea that the clusters of quarks bound in shell model orbits actually have internal structure different from that of a free nucleon. This is addressed in Section 3, where we anticipate the results of a critical experiment performed at Jefferson Lab, which are expected to appear soon. Section 4 summarises this new approach to the structure of the atomic nucleus and looks to further consequences of it.

\section{Nuclear structure: a new force of the Skyrme type}

It is worthwhile to begin with some remarks on the application of effective field theory (EFT) to nuclear structure, since that also is often treated as containing all of the consequences of QCD [15]. Certainly the systematic application of chiral effective field theory to the $N N$ and $N N N$ forces and hence to nuclear structure has proven quite powerful. Such an approach is built upon the symmetries of QCD and is often considered to be equivalent to it. The problem is that the EFT approach needs some power counting scheme, which is a purely human construction. It also needs a set of hadronic degrees of freedom (dof) and that choice too is at the whim of the user. Finally, the EFT typically applied to nuclear problems is non-relativistic.

The usual choice of dof are nucleons and pions. If these are indeed the appropriate dof one is in luck. However, given the remarks in the Introduction, where we saw that on model independent grounds the intermediate range attraction between nucleons is a rather large Lorentz scalar, this is not so obvious. The attractive scalar and repulsive vector forces may cancel (in the central component of the nuclear force) to produce a relatively small amount of binding but the effect of those two components on the internal structure of a nucleon is completely different.

In an EFT the only way to include the effect of a change in the structure of a bound nucleon at the level of QCD is to include nucleon excited states amongst the dof. Typically this is limited to the $\Delta$ resonance, where we do know the relevant couplings quite well. However, given that the $\sigma$ meson has quantum numbers $0^{++}$, one may expect that the inclusion of excitations like the Roper resonance [16] may be relevant. Unfortunately, we have so little knowledge of that state that, at the present time, it would be very difficult to include it in an EFT framework in any reliable manner.

As a consequence, building an EFT of nuclei based upon nucleon and pion dof may not be as accurate an expression of QCD as it may appear at first sight.

An alternative approach to developing an EFT for nuclear structure is based on the density functional approach. There one starts with the QMC model itself and develops a density functional equivalent to it. From this one can use the machinery developed around the Skyrme forces [17], which have proven so successful in the study of both nuclear structure and reactions.

Indeed, using the density functional approach it has proven possible to develop a clear connection between the self-consistent treatment of in-medium hadron structure and the existence of many-body [18] or density dependent [19] effective forces. Dutra et al. [20] critically examined a variety of phenomenological Skyrme models of the effective density dependent nuclear force against the most up-to-date empirical constraints. Amongst the few percent of the Skyrme forces studied which satisfied all of these constraints, the Skyrme model SQMC700, was unique in that it was actually derived from the QMC model and hence incorporated the effects of the internal structure of the nucleon and its modification in-medium. 
Very recently, Stone, Guichon, Reinhard and Thomas [21] carried out a systematic study of the properties of atomic nuclei across the whole periodic table using the new, effective, density-dependent $N N$ force derived from the QMC model [19]. The study began by defining those combinations of the three fundamental couplings in the model (namely the $\sigma, \omega$ and $\rho$ couplings to the up and down quarks) which reproduce the saturation density, binding energy per nucleon and symmetry energy of nuclear matter within the empirical uncertainties on these quantities. Then, a search was carried out for the set of three parameters satisfying this nuclear matter constraint which best described the ground-state properties of a selection of more than 100 nuclei across the entire periodic table.

The root mean-square deviation of the fit from the actual binding energy for this set of nuclei was just $0.35 \%$. For the superheavy nuclei where the binding energies are known, the deviation was a mere $0.1 \%$. This level of agreement with the empirical binding energies is remarkable, in that it is comparable with the very best phenomenological Skyrme forces which have typically 11 or more adjustable parameters.

Not only does this derived effective $N N$ force satisfactorily describe binding energies but going beyond the nuclei used in the fit it accurately describes the evolution of quadrupole deformation across isotopic chains, including shell closures. It also proved capable of describing the observed shape co-existence of prolate, oblate and spherical shapes in the $\mathrm{Zr}$ region. Finally, it naturally gave a double quadrupole-octupole phase transition in the Ra-Th region.

These are remarkable successes given the extremely small number of parameters and this suggests that it would be worthwhile to apply this derived effective force across a variety of challenges in modern nuclear physics.

\section{Experimental tests}

Almost immediately after the creation of the QMC model it was applied [10] to the modification of the valence quark distribution in nuclei discovered by the European Muon Collaboration (EMC), known as the EMC effect [22]. That early work was based on the MIT bag model, for which the calculation of structure functions is possible within some approximations [23] but complicated. More recently, the generalization of the QMC model to the NJL model, suggested by Bentz and Thomas [24], has also been applied to the EMC effect with similar success [25]. The modification of the quark wave functions within the bound nucleons, because of the applied mean scalar field, naturally suppresses the valence distributions at large Bjorken $x$.

While this approach is the only quantitative model of nuclear structure which is able to describe the nuclear EMC effect, it is not yet universally accepted as the explanation for it. For example, it has recently been suggested that the entire EMC effect should be attributed to an as yet uncalculable modification of the nucleons involved in short-range correlations [26], while the rest of the nucleons apparently remain totally unchanged.

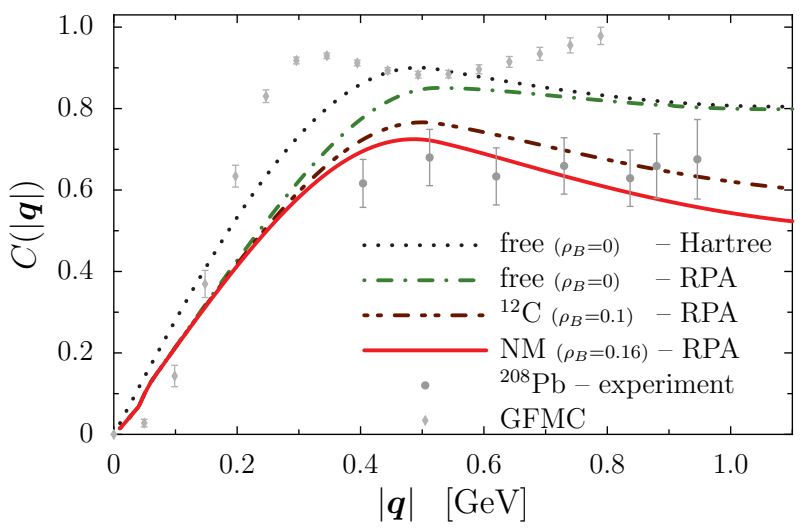

Figure 1. Predictions (from Ref. [36]) for the Coulomb sum rule as a function of three momentum transfer for nuclear matter at densities corresponding to ${ }^{12} \mathrm{C}$ and ${ }^{208} \mathrm{~Pb}$, with or without the effect of the in-medium modification of the nucleon electric form factors. Also shown are the GFMC calculations for ${ }^{12} \mathrm{C}$ (small points [37]) and older experimental data for ${ }^{208} \mathrm{~Pb}[34,38]$.

Another feature of this approach to nuclear structure is that the elastic form factors of the nucleon are also modified in-medium [27]. Using the QMC model, predictions were made almost 20 years ago for the experiment being planned at Jefferson Lab to measure the ratio of the electric to magnetic form factors of a proton bound in ${ }^{4} \mathrm{He}$ [28]. A decade later the measurements were in remarkably good agreement with those predictions [29-31], showing a significant medium modification. However, after the data appeared it was shown that it could also be fit by adding an unusually large polarised charge exchange correction. Although we are aware of no data supporting that proposed correction and no proposal to check it experimentally, it has muddied the waters sufficiently that this cannot yet be regarded as a "smoking gun".

Another suggestion, which seems far less susceptible to unknown nuclear corrections, involves the measurement of the longitudinal response function measured in inelastic electron scattering [34]. That was also examined in the late 90's on the basis of the modification of the electric form factor of the proton [32], already mentioned. Very recently, inspired by the proposal of Meziani and collaborators [33] to make a definitive measurement of this quantity for several nuclei across the periodic table, this response function and the associated Coulomb sum rule of McVoy and van Hove [35] were investigated using the NJL model to describe the structure of both the free and bound nucleons [36]. This work not only treated self-consistently the modification of bound nucleon structure resulting from the mean scalar field but it also included a state-of-the-art treatment of relativistic corrections and RPA correlations. The results are illustrated in Fig. 1.

At high values of the momentum transfer the effect of relativity and of the medium modification of the electric form factor of the proton in particular are both very significant. The older data certainly favours the new calculations and it is clearly vital to have the results of the comprehensive new experiment from Jefferson Lab as soon as 
possible. The beauty of this particular measurement is that it appears to be extremely insensitive to other nuclear corrections, including the effect of short-range correlations.

\section{Summary}

We have presented a compelling argument that within the framework of QCD one is naturally led to the conclusion that the structure of a bound nucleon must differ from that in free space.

This idea has been used to derive, starting from the quark level, a new, density-dependent effective nuclear force which has proven remarkably accurate in describing the properties of finite nuclei across the entire periodic table, while at the same time reproducing the known properties of nuclear matter. We trust that these remarkable results will inspire a great deal more work on nuclear structure within this framework over the coming years.

We have seen that within the quantitative models of nuclear structure that have been developed within this approach, using either the MIT bag or the NJL model to describe nucleon structure, one finds a natural explanation of the nuclear EMC effect. There are also predictions for the modification of the electromagnetic form factors of the bound nucleon, for which the most unambiguous test is the Coulomb sum rule. There is an expectation that definitive new data for this will come from Jefferson Lab in the near future.

Finally, we briefly mention a number of other consequences of this approach to nuclear structure which are both fascinating and the subject of experimental investigation in the near future. For example, a careful study of nuclear structure functions has shown that this approach predicts an important isovector component of the nuclear EMC effect [39]. For a nucleus like ${ }^{56} \mathrm{Fe}$ this leads to a correction to the Paschos-Wolfenstein relation which is of the sign and magnitude to reduce the $\mathrm{NuTeV}$ anomaly by more than one standard deviation. These predictions will be tested directly in future measurements of parity violation [40] at Jefferson Lab following the $12 \mathrm{GeV}$ upgrade.

Within this approach one also finds a remarkably large nuclear modification of the spin dependent parton distributions of the nucleon [41]. Again, future experiments planned at Jefferson Lab will test this through the measurement of the spin structure functions of light nuclei with an unpaired proton.

In conclusion, we stress that while one can derive effective $N N$ forces which can be used in traditional nuclear structure calculations, the underlying physics constitutes a new paradigm for nuclear theory. The quark clusters which occupy shell model orbits in finite nuclei have internal structure which depends on the local scalar field they are not immutable. This simple observation, which is entirely natural within the framework of QCD, explains the saturation of nuclear matter and the nuclear EMC effect and predicts a dramatic reduction in the Coulomb sum rule as well as a multitude of other phenomena which will be subject to experimental study in the coming decade.

\section{Acknowledgements}

I am indebted to the many collaborators who have contributed to the understanding of this approach to nuclear structure, particularly P. A. M. Guichon, W. Bentz, I. Cloët, K. Saito, J. Stone and K. Tsushima. This work was supported by the University of Adelaide and by the Australian Research Council through the ARC Centre of Excellence for Particle Physics at the Terascale (CE110001104), an ARC Australian Laureate Fellowship (FL0992247) and DP150103101.

\section{References}

[1] T. Ueda and A. E. S. Green, Phys. Rev. 174, 1304 (1968).

[2] B. Ananthanarayan, G. Colangelo, J. Gasser and H. Leutwyler, Phys. Rept. 353, 207 (2001).

[3] W. N. Cottingham, M. Lacombe, B. Loiseau, J. M. Richard and R. Vinh Mau, Phys. Rev. D 8, 800 (1973).

[4] J. D. Walecka, Annals Phys. 83, 491 (1974).

[5] B. D. Serot and J. D. Walecka, Phys. Lett. B 87, 172 (1979).

[6] E. D. Bloom et al., Phys. Rev. Lett. 23, 930 (1969).

[7] P. A. M. Guichon, Phys. Lett. B 200, 235 (1988).

[8] A. Chodos, R. L. Jaffe, K. Johnson, C. B. Thorn and V. F. Weisskopf, Phys. Rev. D 9, 3471 (1974).

[9] A. W. Thomas, Adv. Nucl. Phys. 13, 1 (1984).

[10] A. W. Thomas, A. Michels, A. W. Schreiber and P. A. M. Guichon, Phys. Lett. B 233, 43 (1989).

[11] P. A. M. Guichon et al., Nucl. Phys. A 601, 349 (1996).

[12] K. Tsushima et al., Nucl. Phys. A 630, 691 (1998).

[13] K. Tsushima, K. Saito and A. W. Thomas, Phys. Lett. B 411, 9 (1997) [Phys. Lett. B 421, 413 (1998)].

[14] K. Saito, K. Tsushima and A. W. Thomas, Prog. Part. Nucl. Phys. 58, 1 (2007).

[15] S. Weinberg, Nucl. Phys. B 363, 3 (1991).

[16] D. Leinweber et al., arXiv:1511.09146 [hep-lat].

[17] D. Vautherin and D. M. Brink, Phys. Rev. C 5, 626 (1972).

[18] P. A. M. Guichon and A. W. Thomas, Phys. Rev. Lett. 93, 132502 (2004).

[19] P. A. M. Guichon et al., Nucl. Phys. A 772, 1 (2006).

[20] M. Dutra et al., Phys. Rev. C 85, 035201 (2012).

[21] J. R. Stone, P. A. M. Guichon, P. G. Reinhard and A. W. Thomas, Phys. Rev. Lett. 116, 092501 (2016).

[22] J. J. Aubert et al. [European Muon Collaboration], Phys. Lett. B 123, 275 (1983).

[23] A. I. Signal and A. W. Thomas, Phys. Rev. D 40, 2832 (1989).

[24] W. Bentz and A. W. Thomas, Nucl. Phys. A 696, 138 (2001).

[25] I. C. Cloet, W. Bentz and A. W. Thomas, Phys. Lett. B 642, 210 (2006).

[26] D. Wang et al. [PVDIS Collaboration], Nature 506, 67 (2014). 
[27] D. H. Lu, K. Tsushima, A. W. Thomas, A. G. Williams and K. Saito, Phys. Rev. C 60, 068201 (1999).

[28] D. H. Lu, A. W. Thomas, K. Tsushima, A. G. Williams and K. Saito, Phys. Lett. B 417, 217 (1998).

[29] S. Strauch et al. [Jefferson Lab E93-049 Collaboration], Phys. Rev. Lett. 91, 052301 (2003).

[30] M. Paolone et al., Phys. Rev. Lett. 105, 072001 (2010).

[31] J. M. Udias and J. R. Vignote, Phys. Rev. C 62, 034302 (2000).

[32] K. Saito, K. Tsushima and A. W. Thomas, Phys. Lett. B 465, 27 (1999).

[33] Jefferson Lab experiment E05-110: http://hallaweb.jlab.org/experiment/E05110/exp_home/
[34] J. Morgenstern and Z. E. Meziani, Phys. Lett. B 515, 269 (2001).

[35] K. W. McVoy and L. Van Hove, Phys. Rev. 125, 1034 (1962).

[36] I. C. Cloët, W. Bentz and A. W. Thomas, Phys. Rev. Lett. 116, 032701 (2016).

[37] A. Lovato, S. Gandolfi, R. Butler, J. Carlson, E. Lusk, S. C. Pieper and R. Schiavilla, Phys. Rev. Lett. 111, 092501 (2013).

[38] A. Zghiche et al., Nucl. Phys. A 572, 513 (1994) [Nucl. Phys. A 584, 757 (1995)].

[39] I. C. Cloet, W. Bentz and A. W. Thomas, Phys. Rev. Lett. 102, 252301 (2009).

[40] I. C. Cloet, W. Bentz and A. W. Thomas, Phys. Rev. Lett. 109, 182301 (2012).

[41] I. C. Cloet, W. Bentz and A. W. Thomas, Phys. Rev. Lett. 95, 052302 (2005). 